\title{
Anisotropic Interpolation of DT-MRI
}

\author{
Carlos A. Castaño-Moraga ${ }^{1}$, Miguel A. Rodriguez-Florido ${ }^{1}$, Luis Alvarez ${ }^{2}$, \\ Carl-Fredrik Westin ${ }^{3}$, and Juan Ruiz-Alzola ${ }^{1,3}$ \\ 1 Medical Technology Center, Signals \& Communications and Telematic Engineering \\ Departments \\ 2 Informatics and Systems Department \\ Universidad de Las Palmas de Gran Canaria \\ Canary Islands - Spain \\ \{ccasmor,marf, jruiz\}@ctm.ulpgc.es;lalvarez@dis.ulpgc.es \\ http://www.ctm.ulpgc.es \\ 3 Laboratory of Mathematics in Imaging \\ Brigham \& Women's Hospital and Harvard Medical School \\ Boston (MA) - USA \\ westin@bwh.harvard.edu
}

\begin{abstract}
Diffusion tensor MRI (DT-MRI) is an image modality that is gaining clinical importance. After some preliminaries that describe the fundamentals of this imaging modality, we present a new technique to interpolate the diffusion tensor field, preserving boundaries and the constraint of positive-semidefiniteness. Our approach is based on the use of the inverse of the local structure tensor as a metric to compute the distance between the samples and the interpolation point. Interpolation is an essential step in managing digital image data for many different applications. Results on resampling (zooming) synthetic and clinical DTMRI data are used to illustrate the technique. This new scheme provides a new framework for anisotropic image processing, including applications on filtering, registration or segmentation.
\end{abstract}

\section{Introduction}

From its advent in the mid-nineties, diffusion tensor MRI (DT-MRI) has become a powerful imaging modality for many clinical analysis [1]: brain ischemia, multiple sclerosis, leucoencephalopathy, Wallerian degeneration, Alzheimer's disease, white matter fiber tracking, brain connectivity, etc.

This medical image modality provides a discrete volumetric dataset where each voxel contains a second-order positive-semidefinite tensor associated with the effective anisotropic diffusion of water molecules in biological tissues. This information proves very useful, for example, in fiber tractography where the trajectories of nerve tracts and other fibrous tissues are followed by the direction of the eigenvectors associated with the largest eigenvalues of the diffusion tensors [2]. 
A DT-MRI dataset consists of noisy samples drawn from an underlying macroscopic diffusion tensor field, which is assumed continuous and smooth at a coarse anatomical scale. Therefore the measured effective tensor field describes voxel-averaged tensors, and in regions where fibers diverge or converge, bend or twist, branch or merge, there are disparities between the measured macroscopic tensor field and the real heterogeneous microscopic field [3].

In many applications, such as fiber-tract organization or statistical estimation of histological and physiological MRI parameters (e.g. principal diffusivities), it would be desirable to work with higher resolution images. However, a DTMRI dataset usually have low resolution, and upsampling techniques are needed.

DT-MRI upsampling is a problem that has been discussed before. For example, in [4] a continuous tensor field approximation from a discrete set of noisy DT-MRI measurements is proposed. But the upsampled tensor field is not guaranteed to be positive-semidefinite at all points within the imaging volume and the boundaries, where diffusion properties change abruptly, are not preserved.

In this paper we propose a new anisotropic interpolation technique for DTMRI and other positive semidefinite (PSD) tensor data. Interpolation is normally performed by weighting local signal samples according to some rule (linear, cubic, etc.). The idea behind the approach presented here is to weigh the samples in the direction of maximum variation (e.g. across and edge) less than in the orthogonal direction (e.g. along the edge) in order to reduce inter-region blurring. In our scheme this is achieved by using a metric defined from a local structure tensor estimated from tensor data. In the experiments below the local structure tensor is obtained following a recent approach proposed in [5].

Furthermore, since weights naturally are constrained to be positive, if the tensors in the input tensor field are PSD, the interpolated tensors will be PSD as well. It is important to note that this idea can be extended to more general anisotropic tensor estimation problems.

This paper is organized as follows: First, we present the tensor data interpolation problem. Then, we briefly review our recently published approach to tensor coding the local structure of tensor fields, which will prove essential in the new interpolator proposed in this paper and presented in the next section. Finally, illustrative results from synthetic and real DT-MRI data are also presented.

\section{DT-MRI Interpolation}

Interpolating DT-MRI data requires estimating the effective diffusion tensor at unsampled points in order to obtain an upsampled DT-MRI field, with the constraint that the interpolated field is PSD. Of course, it is desirable that bound- 
aries are preserved, something that is not achieved with simple interpolation algorithms.

Our approach to interpolate assumes that the unknown tensor in an upsampled point can be estimated as a linear combination of tensor data sampled from surrounding locations, i.e.,

$$
\hat{\mathbf{D}}(\mathbf{x})=\frac{\sum_{i=1}^{n} \omega_{i} \mathbf{D}\left(\mathbf{x}_{\mathbf{i}}\right)}{\sum_{i=1}^{n} \omega_{i}},
$$

where $\mathbf{x}$ is the interpolation point in our reference system, $\mathbf{x}_{\mathbf{i}}$ the samples position, $n$ the number of samples used to interpolate, and $\omega_{i}$ the weights of those samples. Different interpolators and estimators assign different weights to samples. In some cases it is possible to obtain very efficient separable kernels. Nevertheless, in order to preserve edges, the local structure should be accounted for, and the estimator must be adaptive, with weights changing from one location to another.

Consider for example the weights to be obtained as inversely proportional to the squared distance to the sample,

$$
\omega_{i}=\frac{1}{\left(\mathbf{x}-\mathbf{x}_{\mathbf{i}}\right)^{T}\left(\mathbf{x}-\mathbf{x}_{\mathbf{i}}\right)} .
$$

This inverse squared distance interpolator is usual in many applications with conventional scalar images, but it smooths boundaries and other highly structured regions. If only distance to the sample is accounted for, the estimator mixes samples drawn from areas that can be very different even though they are close, eg. samples from two sides of an edge. Therefore an analysis of the local complexity of the signal is necessary prior to assigning weights to samples in order to avoid this behavior. There are several alternatives to local structure analysis in scalar data, including simple gradient computations, and we have selected the one proposed in [6.7] due to its good properties such as phase invariance and its advantages to gradient measures [7]. This scheme encodes local structure from scalar data in a second order PSD tensor field. Our approach to anisotropic interpolation, and presented for the first time here, makes use of the local structure tensor field to adaptively change the interpolation weights.

In order to interpolate tensor data we follow a similar approach, though now we use a generalization of the previous local structure analysis scheme we published recently for tensor data in [5]. Now, again, local structure is encoded in a second order PSD tensor field.

For the sake of simplicity, and in order to keep the computational burden as low as possible, the interpolation is based on the 4 nearest samples in 2D and on the 8 nearest samples in 3D; this could obviously be extended easily to larger neighborhoods. For regular discrete arrays, such as with DT-MRI data, the interpolated point is located in the center of the pixel/voxel or on an edge/side. Interpolation is carried out in successive sweeps for interpolation in centers, edges and sides. 


\section{Local Structure Estimation in Vector and Tensor Data}

Edges in vector and higher order tensor data (multivalued data) can be defined as regions in the dataset where the signal changes abruptly, and similarly to the scalar case, a local structure tensor can be defined and estimated for such data. As will be described in more detail below, the new interpolation scheme presented in this paper uses the local structure to infer a local metric for the calculation of sample distances.

The local structure tensor proposed by Knutsson [6] is coded by a second order symmetric positive-semidefinite tensor $\mathbf{T}$, represented with respect to a tensor basis $\left\{\mathbf{M}^{k}\right\}: \mathbf{T}=\sum_{k}\left\|q_{k}\right\| \mathbf{M}^{k}$. The coordinates $\left\|q_{k}\right\|$ are the magnitudes of the outputs of a set of multidimensional spherically separable quadrature filters applied to the scalar field, which are maximum in the high structure regions.

The set of quadrature filters is defined in the Fourier domain by:

$$
Q(\boldsymbol{\omega})_{k}= \begin{cases}e^{\left(\frac{-4}{B^{2} \ln 2}\right)\left(\ln ^{2}\left(\frac{\|\boldsymbol{\omega}\|}{\left\|\boldsymbol{\omega}_{o}\right\|}\right)\right)} \cdot\left(\hat{\boldsymbol{\omega}}^{T} \hat{\mathbf{n}}_{k}\right)^{2} & \text { if } \hat{\boldsymbol{\omega}}^{T} \hat{\mathbf{n}}_{k}>0 \\ 0 & \text { otherwise }\end{cases}
$$

where $\hat{\mathbf{n}}_{k}$ is the unitary vector defining the direction of each quadrature filter, and where $\boldsymbol{w}$ denotes the frequency vector. These filters can be seen as an oriented Gaussian function in a logarithmic scale, centered at $\left\|\boldsymbol{\omega}_{o}\right\|$ and with bandwidth $B$. The tensor basis elements $\mathbf{M}^{k}$ are defined by the filter directions $\hat{\mathbf{n}}_{k}$. They are defined as the dual tensor basis to the tensor basis defined by the outer products of these filter directions, $\left\{\hat{\mathbf{n}}_{k} \hat{\mathbf{n}}_{k}^{T}\right\}$. More details about how to calculate the elements $\mathbf{M}^{k}$ can be found in [8].

In order to evenly cover the local spectrum of the dataset, Knutsson proposed to use filter directions defined by the vertexes of the hexagon in $2 \mathrm{D}$, and the icosahedron in 3D.

For an $n$-dimensional scalar dataset, the minimum number of quadrature filters required is $\frac{n(n+1)}{2}$, and the local structure tensor can be written as:

$$
\mathbf{T}=\sum_{k=1}^{n(n+1) / 2}\left\|q_{k}\right\| \mathbf{M}^{k}
$$

Recently we extended the scalar approach to tensor valued data [5] where the local structure tensor is defined by:

$$
\mathbf{T}=\sum_{k=1}^{n(n+1) / 2}\left(\sum_{x y z . . n}\left\|q_{[k] x y z . . n}\right\|^{2}\right)^{\frac{1}{2}} \mathbf{M}^{k}
$$

and subscripts $x y z . . n$ are associated to the components of the tensor field.

\section{Anisotropic Interpolation Weighted by Local Structure}

The local structure tensor field consists of a field of second-order symmetric PSD tensors, which can be associated with ellipsoids. These ellipsoids tend to be big 


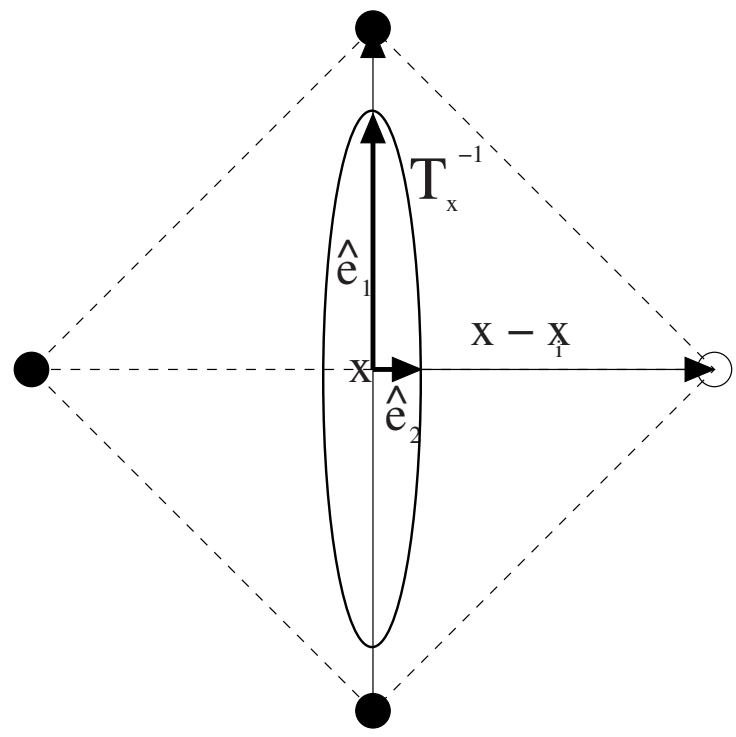

Fig. 1. Regular grid with tensors from different regions represented as white and black dots, respectively.

when there are steep changes in the signal and small otherwise. Moreover, the ellipsoids are elongated in the direction of maximum signal variation. Rounder tensors either mean two or more directions of signal variation (e.g. a corner) or no significant direction of variation (e.g. homogeneous signal). The local structure tensor can therefore be used to control how samples are combined when building signal estimators and, in particular, samples on two different sides of a strong edge should not be combined.

In this work, we propose to use the inverse of the local structure tensor as a metric tensor. The inversion corresponds to a $\pi / 2$ radians rotation of the associated ellipsoid, and it is necessary to weigh the samples along the direction of maximum signal variation less than those in the orthogonal one. This is illustrated in Fig. 1, where tensors from different regions are represented as black and white dots in a regular grid. As it can be seen, although the distance between the interpolated point and data samples is the same, their contributions are not.

Hence, the weights for the linear combination in (1) are computed as

$$
\omega_{i}=\left(\mathbf{x}-\mathbf{x}_{\mathbf{i}}\right)^{T} \mathbf{T}_{\mathbf{x}}^{-\mathbf{1}}\left(\mathbf{x}-\mathbf{x}_{\mathbf{i}}\right),
$$

where $T_{\mathbf{x}}$ denotes the local structure tensor at the interpolation point.

Since these weights are positive (the metric tensors are positive semidefinite), the interpolated tensors are PSD if input tensors are. 

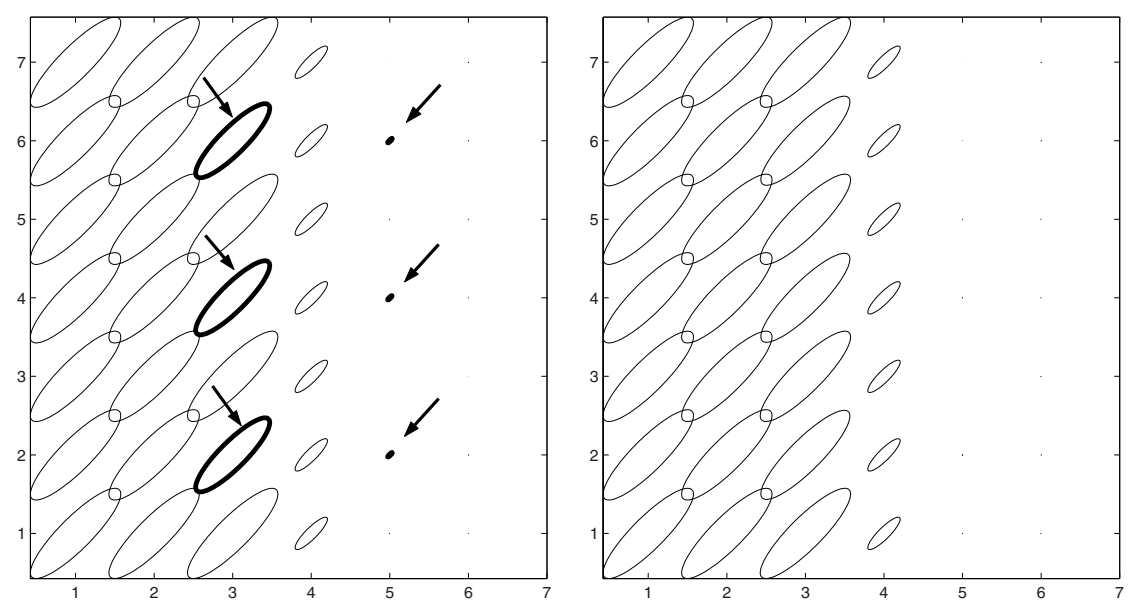

Fig. 2. Results from interpolation of tensor valued data using linear interpolation (left), and anisotropic interpolation (right).

\section{Results}

In this section, we present some experiments with synthetic and clinical DT-MRI data.

Synthetic Data. To assess our approach in the presence of edges, we perform interpolation of a tensor field consisting of a step edge with constant tensors on one side and zero tensors on the other side. In figure 2 we compare interpolation using linear interpolation (left) with the approach presented in this paper (right). To estimate the local structure tensor field with (5), we use the values $\left\|\omega_{0}\right\|=\frac{\pi}{8}$, and $B=2$ in (3). The quadrature filters are oriented in the directions pointing to the vertexes of a hexagon. Notice that the edge is smeared out further using linear interpolation compared to the proposed method using the inverse structure tensor as a local metric.

DT-MRI Data. The DT-MRI data was obtained at Brigham \& Women's Hospital (Boston (MA) - USA) from a GE Signa 1.5 Tesla Horizon Echospeed 5.6 system with standard 2.2 Gauss/cm field gradients. The voxel resolution is $0.85 \mathrm{~mm} \times 0.85 \mathrm{~mm} \times 5 \mathrm{~mm}$.

A central area in an axial slice (see Fig. 3 left) is then zoomed (right). Again edges are correctly preserved by the interpolation algorithm.

\section{Conclusions}

Interpolation is essential for many important tasks managing digital imagery and, in particular for data of low resolution such as clinical DT-MRI data. In 

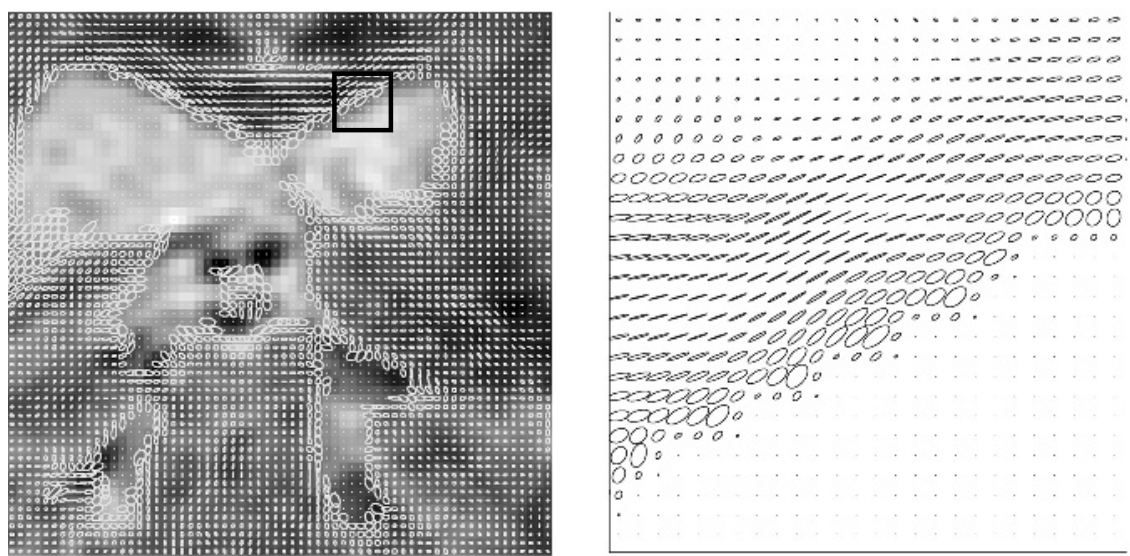

Fig. 3. DT-MRI $64 \times 64$ slice of the corpus callosum superposed over the corresponding MRI T2 image (left) and a zoomed part of the central area (right).

this paper we present a new method to interpolate a tensor field, preserving boundaries and assuring the positive-semidefinite constraint. Our approach interpolates tensors by using the natural neighbors of the interpolation point, in a regular grid, with weights proportional to the inverse squared distance between the samples and the interpolation point, using the inverse of a generalization of the local structure tensor as a metric to compute the distance.

This scheme has been applied to synthetic and in vivo DT-MRI data with very encouraging results.

The ideas presented in this work can be extended to more general problems involving anisotropic estimation from digital image data (scalar, vector, and tensor data). We are currently working on such extensions.

Acknowledgements. This work was supported by a FPU grant at the Spanish Ministry of Education AP2002-2774, the Spanish Ministry of Science and Technology and European Commission, co-funded grant TIC-2001-38008-C02-01, and the US grants NIH P41-RR13218 and CIMIT.

\section{References}

1. Le Bihan D., Mangin J.-F., Poupon C., Clark C.A., Pappata S., Molko N. and Chabriat H.: Diffusion tensor imaging: Concepts and applications. Magn. Reson. Imaging J. 13 (2001) 534-546

2. Basser P.J., Pajevic S., Pierpaoli C. and Aldroubi A.: Fiber tract following in the human brain using DT-MRI data. IEICE Trans. Inf. \& Sist. 1 (2002) 15-21

3. Pierpaoli C. and Basser P.J.: Toward a quantitative assesment of diffusion anisotropy. Magn. Reson. Med. 36 (1996) 893-906 
4. Pajevic S., Aldroubi A., and Basser P.J.: A continuous tensor field approximation of discrete DT-MRI data for extracting microstructural and architectural features of tissue. Journal of Magnetic Resonance 154 (2002) 85-100

5. Rodriguez-Florido M.A., Westin C.-F., and Ruiz-Alzola J.: DT-MRI regularization using anisotropic tensor field filtering. In: 2004 IEEE International Symposium on Biomedical Imaging - April 15-18 - Arlington,VA (USA). (2004) 336-339

6. Knutsson, H.: Representing local structure using tensors. In: 6th Scandinavian Conference on Image Analysis. Oulu, Finland. (1989) 244-251

7. Knutsson H., and Andersson M.: What's so good about quadrature filters? In: 2003 IEEE International Conference on Image Processing. (2003)

8. Westin, C.F., Richolt, J., Moharir, V., Kikinis, R.: Affine adaptive filtering of CT data. Medical Image Analysis 4 (2000) 161-172 